OPEN

SUBJECT AREAS:

NANOSCIENCE AND

TECHNOLOGY

THERMOELECTRICS

SYNTHESIS

NANOSCALE MATERIALS

Received

15 October 2012

Accepted

21 November 2012

Published

11 December 2012

Correspondence and requests for materials should be addressed to

S.-H.Y. (shyu@ustc. edu.cn)

\section{Linearly arranged polytypic CZTSSe nanocrystals}

\author{
Feng-Jia Fan' ', Liang Wu', Ming Gong ${ }^{2}$, Shi You Chen ${ }^{3}$, Guang Yao Liu ${ }^{4}$, Hong-Bin Yao' , Hai-Wei Liang', \\ Yi-Xiu Wang' \& Shu-Hong Yu'
}

'Division of Nanomaterials \& Chemistry, Hefei National Laboratory for Physical Sciences at Microscale, Department of Chemistry, CAS Key Laboratory of Mechanical Behavior and Design of Materials, the National Synchrotron Radiation Laboratory, University of Science and Technology of China, Hefei 230026, P. R. China, ${ }^{2}$ Engineering and Materials Science Experiment Center, University of Science and Technology of China, Hefei 230026, P. R. China, ${ }^{3}$ Laboratory of Polar Materials and Devices, East China Normal University, Shanghai 200241, China, ${ }^{4}$ Beijing National Laboratory for Condensed Matter Physics, Institute of Physics, Chinese Academy of Sciences, Beiijing 100190, China.

Even colloidal polytypic nanostructures show promising future in band-gap tuning and alignment, researches on them have been much less reported than the standard nano-heterostructures because of the difficulties involved in synthesis. Up to now, controlled synthesis of colloidal polytypic nanocrsytals has been only realized in II-VI tetrapod and octopod nanocrystals with branched configurations. Herein, we report a colloidal approach for synthesizing non-branched but linearly arranged polytypic $\mathrm{I}_{2}$-II-IV-VI $\mathrm{I}_{4}$ nanocrystals, with a focus on polytypic non-stoichiometric $\mathrm{Cu}_{2} \mathrm{ZnSnS}_{\mathrm{x}} \mathrm{Se}_{4-\mathrm{x}}$ nanocrystals. Each synthesized polytypic non-stoichiometric $\mathrm{Cu}_{2} \mathrm{ZnSnS}_{\mathrm{x}} \mathrm{Se}_{4-\mathrm{x}}$ nanocrystal is consisted of two zinc blende-derived ends and one wurtzite-derived center part. The formation mechanism has been studied and the phase composition can be tuned through adjusting the reaction temperature, which brings a new band-gap tuning approach to $\mathrm{Cu}_{2} \mathrm{ZnSnS}_{\mathrm{x}} \mathrm{Se}_{4-\mathrm{x}}$ nanocrystals.

2 (1) olytypism widely exists in IV, III-V and II-VI semiconductors due to their large atom stacking freedom and the most commonly observed polymorphs are zinc blende (ZB) and wurtzite (WZ) ${ }^{1-3}$. The energy gap between different polymorphs, which depends on the ionicity of the chemical bonding ${ }^{4}$, could be overcome during the materials synthesis ${ }^{5}$. Furthermore, since the electronic band structure is correlated to the crystal structure of material, polymorphs with the same chemical composition behave differently in optical, electronic and other properties ${ }^{3,6-8}$. Therefore, the uncontrollable defect of random polytypism introduced in the synthesis stage would have some uncertain effects on the properties of the materials ${ }^{9,10}$. Recently, controlled polytypism and twinning engineering in one single III-V nanowire have been realized in vapor-liquid-solid (VLS) growth ${ }^{9-11}$, and these engineered structure defects are expected to be useful in future optical, electronic and photovoltaic devices, as the variations in electron band structure of different polymorphs provide new possibilities to the band-gap tuning and alignment of semiconductor materials $s^{12,13}$.

In solution based colloidal approach, although the synthesis of standard-heterostructured nanocrystals ${ }^{14-16}$, in which no less than two compounds are incorporated, has been well developed, the controlled engineering of different polymorphs in one single nanostructure has been less reported due to the difficulty in precise phase control. Colloidal synthesis of branched polytypic II-VI nanocrystals with ZB cores and epitaxially grown WZ arms, such as $\mathrm{CdSe}^{17,18}$ and $\mathrm{CdTe}^{19}$ tetrapod nanocrystals, are the only few examples of controlled polytypism engineering in single nanoparticles. Branched polytypic nanocrystals can have up to eight arms, because there are eight $\{111\}_{\mathrm{ZB}}$ facets in the ZB cores can interface with $\{0002\}_{\mathrm{WZ}}$ facets of the WZ arms ${ }^{20}$, even though configurations with four arms in branched nanocrystals are more commonly observed. For those polytypic nanocrystals nucleating in WZ phase and growing in ZB phase, two polymorphs will be arranged in a linear fashion, as there are two opposite $\{0002\}_{\mathrm{WZ}}$ facets. However, to the best of our knowledge, controlled synthesis of this kind of linearly arranged polytypic nanostructure in solution has not been reported so far.

In this contribution, we report our recent accomplishment on the controlled colloidal synthesis of linearly arranged ZB-/WZ-/ZB-derivative polytypic semiconductor nanocrystals with focusing on non-stoichiometric $\mathrm{Cu}_{2} \mathrm{ZnSnS}_{\mathrm{x}} \mathrm{Se}_{4-\mathrm{x}}$ (CZTSSe). We choose CZTSSe to descript the structure features, formation mechanism and phase ratio tuning process, as $\mathrm{Cu}_{2} \mathrm{ZnSnS}_{4}{ }^{21-23}$ (CZTS), $\mathrm{Cu}_{2} \mathrm{ZnSnSe}_{4}{ }_{4}^{24}$ (CZTSe) and their alloy CZTSSe es "nanocrystals ink" are drawing increasing attentions due to their promising applications in future low-cost thin film solar cells ${ }^{26}$. We also demonstrate that the method developed here can be generally applied to synthesize 
$\mathrm{Cu}_{2} \mathrm{CdSnS}_{\mathrm{x}} \mathrm{Se}_{4-\mathrm{x}}$ (CCTSSe) polytypic nanocrystals, of which the major content $\mathrm{Cu}_{2} \mathrm{CdSnSe}_{4}$ (CCTSe) is also an important quaternary chalcogenide due to its potential thermoelectric applications ${ }^{27-30}$.

\section{Results}

Characterization of polytypic CZTSSe nanocrystals. Similar with ternary $\mathrm{CuInS}_{2}$ (CIS) and $\mathrm{CuInSe}_{2}$ (CISe), CZTSe quaternary semiconductors can be taken as derived from the binary $\mathrm{ZnSe}$ through replacing part of $\mathrm{Zn}$ atoms by $\mathrm{Cu}$ and $\mathrm{Sn}$ atoms (see Supplementary Table S1 online). As we known, the binary ZnSe have the ZB and WZ structures, so CIS, CISe and CZTSe have the corresponding ZB-derived and WZ-derived structures. The ground states of the above mentioned ternary and quaternary chalcogenides are the $\mathrm{ZB}$-derived phases, but recent reports have demonstrated that the metastable WZ-derived $\mathrm{CuInS}_{2}{ }^{31}$ and $\mathrm{CuInSe}_{2}{ }^{32}$ nanocrystals can be prepared by using dodecanethiol and diphenyl diselenide, respectively, and two more recent works have demonstrated that WZderived $\mathrm{CZTS}^{33}$ and $\mathrm{CZTSe}^{34}$ can also be prepared through using dodecanethiol and diphenyl diselenide, respectively. Furthermore, unexpected polytypism has been found in CIS nanodiscs, where the WZ phase interfaces with significant chalcopyrite domains ${ }^{35}$.

In our experiments, linear arrangement of ZB- and WZ-derived CZTSSe in single nanoparticle can be achieved in the presence of both dodecanethiol and diphenyl diselenide. The samples of the obtained polytypic CZTSSe nanocrystals were characterized by the powder X-ray diffraction (PXRD) pattern (Fig. 1). For comparison, the X-ray diffraction (XRD) patterns of simulated ZB-derived and WZ-derived CZTSe structures are also presented (see Supplementary Fig. S1 online). Rietveld refinement analysis is performed and the result indicates that the obtained product is actually composed of two phases, i.e. WZ- and ZB-derivatives, with a phase composition of $35.03 \pm 0.41 \%$ for $\mathrm{ZB}$-derived structure. It is rather difficult to distinguish further the precise cation occupations of the obtained CZTSSe nanocrystals, because different polymorphs derived from the same structure show almost the same PXRD patterns. Considering ZBand WZ-derived structures possess similar anion matrix to binary $\mathrm{ZB}$ and $\mathrm{WZZ} \mathrm{ZnSe}$, respectively, we use the same notation for the crystallographic facets and directions of CZTSSe nanocrystals as those of ZnSe. To avoid ambiguity, four-index Miller-Bravais notation is used to name crystallographic facets and directions in WZ-derived structures.

The morphology of the synthesized polytypic CZTSSe nanocrystals was characterized by transmission electronic microscope (TEM). A typical bright-field TEM image in Fig. 2a shows that the synthesized nanocrystals have uniform rugby-like morphology, of which the long and short diameters are $22 \pm 4 \mathrm{~nm}$ and $13 \pm 2 \mathrm{~nm}$, respectively. The bright-field TEM images of two single nanocrystals in Fig. $2 b, 2 d$ show that contrasts of different domains in the single

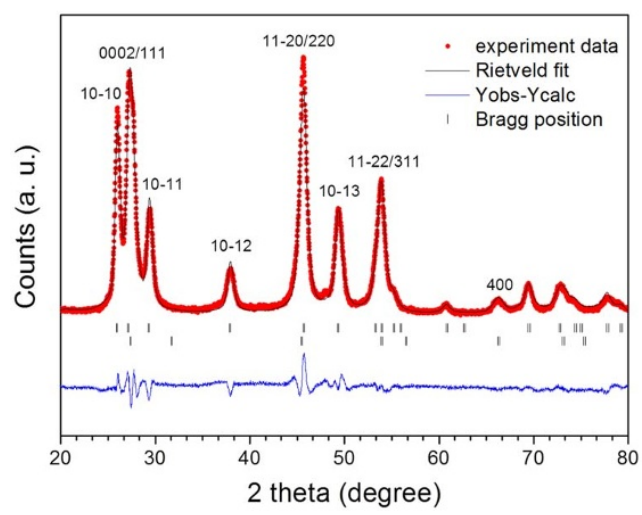

Figure 1 PXRD pattern and Rietveld fit of the obtained polytypic CZTSSe nanocrystals (Rwp: $10.6 \%$, Rexp: $3.24 \%$ and $\chi^{2}: 10.8 \%$ ).

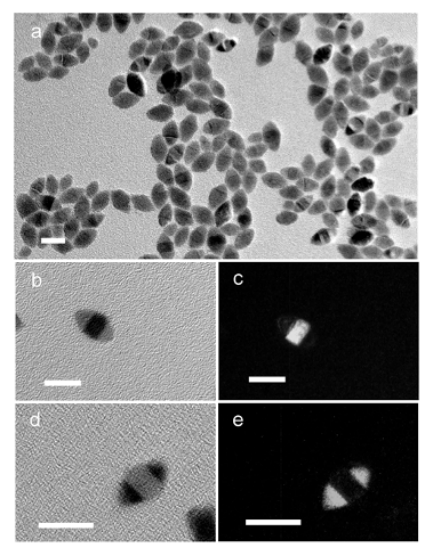

Figure $2 \mid$ Bright-field and dark-field TEM images of polytypic CZTSSe nanocrystals. (a) A typical bright-field TEM image of the synthesized polytypic CZTSSe nanocrystals. (b) and (c) bright-field and the corresponding dark-field TEM images of one individual polytypic CZTSSe nanocrystal. (c) and (d) bright-field and the corresponding dark-field TEM images of another single polytypic CZTSSe nanocrystal. The scale bars are all $20 \mathrm{~nm}$.

nanocrystals are totally different, i.e., two ends show similar contrasts while the central parts differ obviously. The contrast difference can be more clearly observed in the corresponding dark-field TEM images (Fig. 2c, 2e). For some nanocrystals represented in Fig. 2a, although the contrast differences are not obvious, the crystalline boundaries separating nanocrystals into three domains are clearly observed. Noting that the TEM observation of contrast difference and crystalline boundaries are affected by the orientations of the nanocrystals which were randomly deposited on the TEM grid, we tilted the sample within angles of $\pm 15^{\circ}$ along the $x$ and $y$-axis of the TEM grid holder during the observation and found the contrast difference could be observable or absent in some nanocrystals with certain tilting angles. According to these TEM images with different tilting angles (see Supplementary Fig. S2 online), we found about $60 \%$ nanocrystals crystallized with ZB-/WZ-/ZB-derived polytypic nanostructure, it is possibly that the actual proportion of ZB-/WZ-/ ZB-derivative polytypic nanocrystals is even higher, as contrast difference of some nanocrystals might be observable in certain angles which have not been reached in our observation.

As we know, the contrast in bright-field TEM image is correlated to the mass-thickness and diffraction. Mass-thickness contrast is very common in standard nano-heterostructures, in which heavy atoms are usually darker than light ones ${ }^{36}$. The energy dispersive spectroscopy (EDS) analysis firstly confirmed that the average chemical composition of nanocrystals is $\mathrm{Cu}_{2} \mathrm{Zn}_{0.5} \mathrm{Sn}_{0.9} \mathrm{Se}_{2.8} \mathrm{~S}_{0.3}$ (see Supplementary Fig. S3 and Table S2). Noting that the molar ratio of cation precursors is kept as the stoichiometric value, $\mathrm{Zn}$ deficiencies here can be attributed to the lower reactivity of $\mathrm{Zn}$ precursor ${ }^{37}$. To clarify whether the contrast difference is attributed to the variation in composition, high resolution scanning transmission electron microscope energy dispersive spectrometer (STEM-EDS) elemental mapping was then used to characterize whether five elements are non-uniformly distributed in obtained nanocrystals. As shown in Fig. 3, Cu, Zn, Sn, S and Se atoms distribute homogeneously in three nanocrystals and no noticeable nano-heterostructure can be observed in the mapping. Furthermore, an EDS line scan analysis further confirms the uniform distribution of elements (see Supplementary Fig. S4 online). Therefore, we can confirm that the contrast difference in those nanocrystals is aroused from the variation in diffraction, which is caused by the difference in crystalline structures or the orientation between the two ends and the center part of those nanocrystals. 

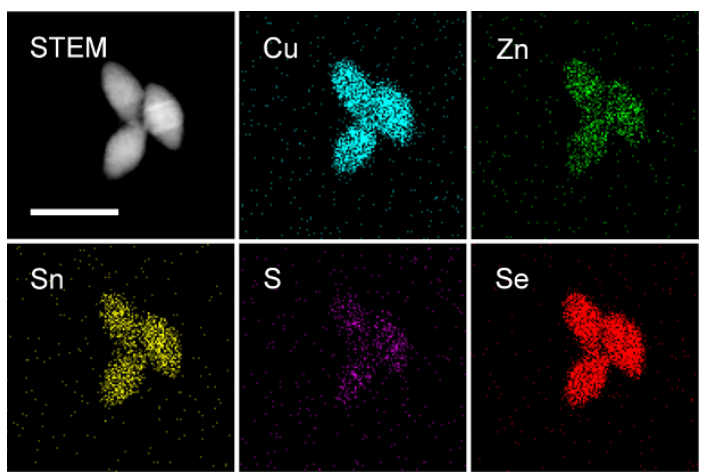

Figure 3 STEM image and high resolution STEM-EDS elemental mappings of three polytypic CZTSSe nanocrystals. The scale bar in STEM image is $30 \mathrm{~nm}$.

High resolution transmission electron microscope (HRTEM) and the corresponding fast Fourier transform (FFT) images are shown in Fig. $4 \mathrm{a}, 4 \mathrm{~b}, 4 \mathrm{c}$ and $4 \mathrm{~d}$ in order to study the detailed crystalline feature of such polytypic nanocrystals. In accordance with the observation on TEM images, the crystalline domain of nanocrystal can be divided into three distinct parts, the crystalline phase of the two ends is ZBderived structure and the center part is WZ-derived structure. A magnified HRTEM image and the corresponding crystal model (Fig. 4e, 4f) show the interface of those two structures where we can observe the lattice mismatch induced stress. The ZB-derived and the corresponding WZ-derived structures differ in their anion atom stacking sequence along $[111]_{\mathrm{ZB}}$ and $[0001]_{\mathrm{WZ}}$ directions. The anion stacking sequence of $\mathrm{ZB}$-derived structure is $\cdots \mathrm{ABCABC} \cdots$ while for WZ-derived structure it is $\cdots \mathrm{ABAB} \cdots$. The stacking in asobtained polytypic nanocrystals here have two possible typical sequences, $\cdots \mathrm{ABC} / \mathrm{AB} \cdots \mathrm{AB} / \mathrm{CBA} \cdots$ and $\cdots \mathrm{ABC} / \mathrm{AB} \cdots \mathrm{AB} / \mathrm{ABC} \cdots$, both of them have been observed in the HRTEM images and two typical HRTEM images are represented in Fig. $4 \mathrm{a}$ and $4 \mathrm{~b}$. In the former case (Fig. 4a), the stacking orders of two ends are opposite and the central WZ-derived domain just acts as a twinning plane of ZB-derived structure; in the latter case (Fig. 4b), it looks like that one $\mathrm{ZB}$-derived nanoparticle is separated by a WZ-derived domain.

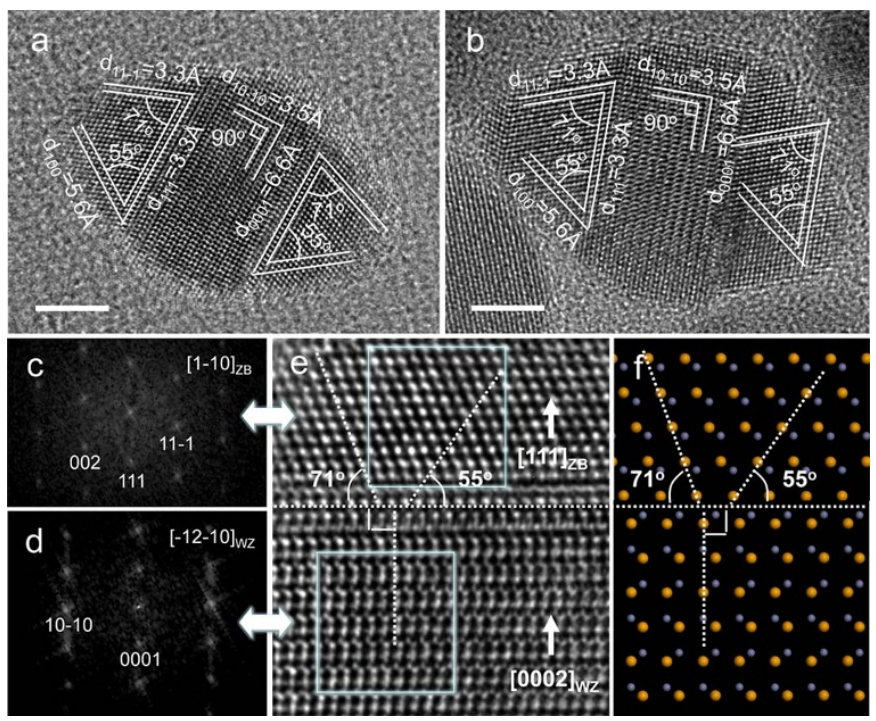

Figure $4 \mid$ High resolution TEM images and the corresponding FFT images. (a) and (b) HRTEM images of two typical polytypic CZTSSe nanocrystals. (c) and (d) FFT images of different area in HRTEM image (e). (f) the crystal model of the interface of ZB- and WZ-derived structures, the orange and dark blue balls represent cation and anion atoms respectively. The scale bars in HRTEM images are both $5 \mathrm{~nm}$.

\section{Discussion}

Time dependent experiments were performed to study the growth process of these novel polytypic nanocrystals. According to the color change of solution during the reaction, the growth of nanocrystals should start at about $220^{\circ} \mathrm{C}$, so we quenched the reaction when the reaction temperature increased to 240,260 and $280^{\circ} \mathrm{C}$, respectively. The intermediate products were characterized by PXRD, TEM, and HRTEM analysis. The majority of atoms in nanocrystals crystallize in WZ-derived structure when the reaction temperature increases to $240^{\circ} \mathrm{C}$ (see Supplementary Fig. S5 online), as the diffraction intensity of $(0020)_{W Z}$ facet, which overlaps with the strongest $(111)_{\mathrm{ZB}}$ diffraction peak of $\mathrm{ZB}$-derived structure, is pretty weak. The phase composition of $\mathrm{ZB}$-derived structure calculated from Rietveld refinement analysis is $6.49 \pm 0.42 \%$ (see Supplementary Fig. S5 and Table S3 online). A HRTEM image taken along $[1-210]_{\mathrm{WZ}}$ direction viewing from the side of one single nanocrystal also indicates that ZB-derived structure can be hardly found (see Supplementary Fig. S6a). Relative diffraction intensities of $(0002)_{\mathrm{WZ}} /(111)_{\mathrm{ZB}}$ and $(11-20)_{\mathrm{wZ}} /(220)_{\mathrm{ZB}}$ facets in PXRD pattern of product obtained at $260^{\circ} \mathrm{C}$ are stronger than those of the product obtained at $240^{\circ} \mathrm{C}$, indicating an increased ZB-derived phase composition (17.62 $\pm 0.71 \%$, see Supplementary Fig. S5 and Table S3). This is consistent with the TEM and HRTEM observation (see Supplementary Fig. S6b), where we could notice that ZB-derived phase grows epitaxially on WZ-derived structure, resulting oval-like structures. When the reaction temperature further increases to $280^{\circ} \mathrm{C}$, the $\mathrm{ZB}$-derived phase composition increases to $27.76 \pm 0.55 \%$ (see Supplementary Fig. S5 and Table S3). The increment of ZB-derived structure in two ends of nanocrystals can also be directly observed in TEM and HRTEM images (see Supplementary Fig. S6c).

To clarify whether the ZB-derived CZTSSe ends are transformed from the metastable WZ-derived CZTSSe cores, we further annealed the nanoparticles deposited on labeled copper TEM grids to test the phase stability of WZ-derived CZTSSe. After being heated at $300^{\circ} \mathrm{C}$ for $10 \mathrm{~min}$, there is no noticeable change in the core size of nanocrystal (see Supplementary Fig. S8 online), and thus we can conclude that the ZB-derived CZTSSe ends mainly result from the nucleation of $\mathrm{ZB}$-derived structure at relative high temperature.

According to the above analysis, we can conclude that these linearly arranged polytypic CZTSSe nanocrystals result from the nucleation in the WZ-derived structure at relative low temperatures with subsequent epitaxial growth of $\mathrm{ZB}$-derived structure at relative high temperatures. To engineer two phases with a controlled manner is an critical issue for the synthesis of polytypic nanocrystals, in our experiments, diphenyl diselenide plays an important role in the formation of metastable WZ-derived phase at relative low temperatures as reported previously in the synthesis of wurtzite CISe nanocrystals $^{32}$, and it also works as selenium source for the growth of stable ZB-derived phase at relative high temperatures.

Based on the understanding of the growth mechanism, we successfully prepared ZB-/WZ-/ZB-derivative polytypic nanocrystals with different phase ratios by changing the reaction temperature from 240 to $320^{\circ} \mathrm{C}$ while the reaction time was all kept at 1 hour. As Fig. 5a, b shows, the nanocrystals obtained after reaction at $240^{\circ} \mathrm{C}$ represent quasi-sphere shapes, and a HRTEM image shows that the WZ-derived structure is the dominant phase. The average aspect ratio of nanocrystals and the $\mathrm{ZB}$-derived phase composition increases as the reaction temperature increases from 240 to $320^{\circ} \mathrm{C}$, and the dominant phase of those nanocrystals obtained at $320^{\circ} \mathrm{C}$ converts to ZB-derived structure (Fig. 5g, h).

The proportions of WZ-derived and ZB-derived structures in the obtained nanocrystals were calculated by using Rietveld refinement analysis (see Supplementary Fig. S7 and Table S4 online). The results are listed in Table 1, the correlations between the composition of ZBderived structure and reaction temperature are consistent with the results observed from the TEM and HRTEM images (Fig. 5): the 


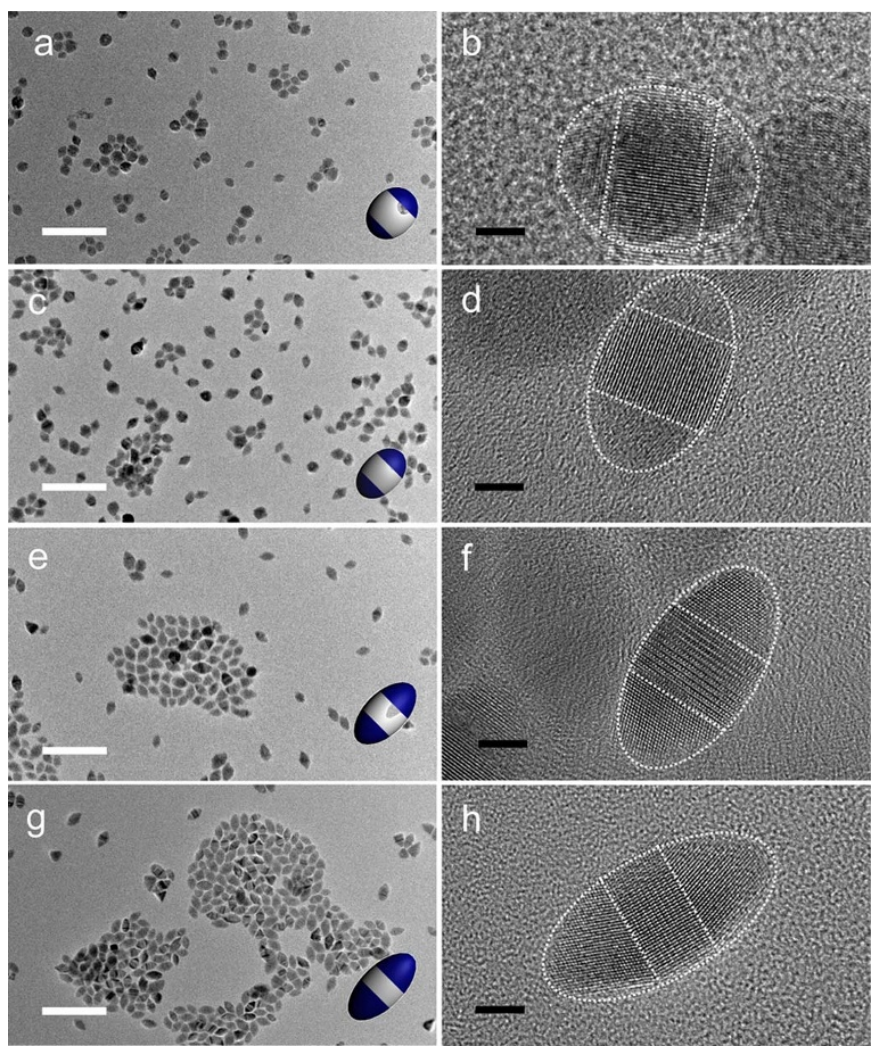

Figure 5 | Typical TEM and HRTEM images of the synthesized polytypic CZTSSe nanocrystals obtained after reaction at different temperatures for $1 \mathrm{~h}$. (a) and (b) $240^{\circ} \mathrm{C}$, (c) and (d) $260^{\circ} \mathrm{C}$, (e) and (f) $300^{\circ} \mathrm{C}$, (g) and (h) $320^{\circ} \mathrm{C}$. The white scale bars are $100 \mathrm{~nm}$ and black scale bars are $5 \mathrm{~nm}$. Insets show the model illustration of the polytypic nanocrystal with different phase composition.

calculated phase compositions of $\mathrm{ZB}$-derived structure in product synthesized at $240,260,300$ and $320^{\circ} \mathrm{C}$ are $19.34 \pm 0.43 \%, 32.70 \pm$ $0.40 \%, 43.62 \pm 0.38 \%$ and $54.67 \pm 0.33 \%$, respectively. It further confirms our proposed formation mechanism that CZTSSe nanocrystals favor WZ-derived structure at relatively low temperatures and then tend to crystallize in $\mathrm{ZB}$-derived structure at relatively high temperatures.

EDS analysis was used to characterize the average chemical compositions of nanocrystals obtained at different temperatures (see Supplementary Fig. S3 and Table S2 online), and UV-vis-NIR spectra (see Supplementary Fig. S9 and Fig. S10 online) were applied to estimate band-gaps through extrapolating the $(\alpha h v)^{2}$ versus photon energy to low energy. It has been noted that all five samples show almost the same molar ratio of Se/S with slight variation, this kind of slight variation is common in our observations but doesn't dependent on the sizes of the nanoparticles. The evaluated band-gaps of those nanocrystals synthesized at $260^{\circ} \mathrm{C}, 280^{\circ} \mathrm{C}$ and $300^{\circ} \mathrm{C}$ are close to each other (Table 1) and can't be distinguished by the estimation methods here, this is reasonable as they have similar compositions and close phase compositions of ZB-derived structure. For the nanocrystals synthesized at $240^{\circ} \mathrm{C}$ have similar compositions to those nanocrystals mentioned above while showing a smaller band-gap, indicating the phase ratio of polytypic nanocrystals would have certain effect on the band-gap. Previous research found that the bandgap of GaAs nanowire could be tuned from $1.515 \mathrm{eV}$ down to $1.43 \mathrm{eV}$ by increasing the phase composition of $\mathrm{WZ}$ structure to $\mathrm{ZB}$ structure, and carrier confinement induced by the band offset staging between $\mathrm{ZB}$ and $\mathrm{WZ}$ structure was used to explain that phenomenon ${ }^{38}$. The smaller band-gap of nanocrystals synthesized at $240^{\circ} \mathrm{C}$ could also be understood by the similar mechanism, as the theoretical calculation has shown that WZ-derived and ZB-derived CZTSSe differ slightly in their band structure ${ }^{39}$. We have not made a comparison between nanocrystals synthesized at $320^{\circ} \mathrm{C}$ and other nanocrystals, because they have different chemical compositions.

It has been predicted that the type-II band-gap alignment exists on the $\mathrm{WZ} / \mathrm{ZB} \mathrm{CdX}\left(\mathrm{X}=\mathrm{S}\right.$, Se and Te) interface ${ }^{40}$, and it also happens on the interface of different $\mathrm{GaInP}_{2}$ polytypes $^{13}$. For our polytypic CZTSSe nanocrystals, the type-II band-gap alignment could be expected on the interfaces between the ZB-derived and WZ-derived structures $^{39}$, which can facilitate the electron-hole separation in the nanocrystals and is a positive factor for their potential photovoltaic applications. Adjusting the proportion of two structures offers a pathway to the band-gap tuning, thus yielding more avenues to optimize the performance of polytypic nanocrystal incorporated devices.

It should be pointed out that the method developed here is not only restricted to the synthesis of polytypic CZTSSe nanocrystals. We found that $\mathrm{Cu}_{2} \mathrm{CdSnS}_{\mathrm{x}} \mathrm{Se}_{4-\mathrm{x}}$ (CCTSSe) polytypic nanocrystals, of which the major content is an important semiconductor due to its potential applications in photovoltaic and thermoelectric devices ${ }^{27-30}$, can be also prepared through a similar procedure (see Supplementary Figs. S11S15 online). This also indicates that the present synthetic principle could be applied to other polytypic semiconductor nanocrystals.

In summary, we have developed an efficient method to synthesize linearly arranged polytypic nanocrystals with focusing on CZTSSe polytypic nanocrystal. PXRD, TEM, HRTEM, EDS, STEM-EDS-elemental mapping and EDS-line scan were used to characterize the obtained CZTSSe polytypic nanocrystals. The as-prepared nanocrystals with rugby-like shape have three separated crystalline domains, two ends of nanocrystals crystallized in ZB-derived phase and the central part grew in WZ-derived structure. The formation mechanism of these CZTSSe nanocrystals has been studied through quenching the reaction during the temperature ramping process. We find the CZTSSe nanocrystals prefer to nucleate in WZ-derived structure at relatively low temperatures while tend to grow with ZBderived structure at high temperatures. Based on that, we successfully prepared polytypic CZTSSe nanocrystals with ZB-derived phase proportions ranging from $19.34 \%$ to $54.67 \%$ through tuning the reaction temperatures from $240^{\circ} \mathrm{C}$ to $320^{\circ} \mathrm{C}$. Furthermore, linearly arranged polytypic CCTSSe can be also prepared through a similar procedure, implying that the synthetic principle of polytypic nanocrsytals reported here could also be applied to other semiconductors that have WZ- and ZB-derived structures and the growth of different polymorphs is sensitive to the reaction temperature. The polytypic

Table 1 | Correlations among reaction temperature, ZB-derived phase composition, chemical composition and band-gap of the polytypic CZTSSe nanocrystals

Reaction temperature $\left({ }^{\circ} \mathrm{C}\right)$ ZB-derived phase composition (\%)

Chemical composition

$\mathrm{Cu}_{2} \mathrm{Zn}_{0.5} \mathrm{Sn}_{0.9} \mathrm{~S}_{0.3} \mathrm{Se}_{2.8}$

$\mathrm{Cu}_{2} \mathrm{Zn}_{0.5} \mathrm{Sn}_{0.9} \mathrm{~S}_{0.3} \mathrm{Se}_{3.0}$

$\mathrm{Cu}_{2} \mathrm{Zn}_{0.5} \mathrm{Sn}_{0.9} \mathrm{~S}_{0.3} \mathrm{Se}_{2.8}$

280

300

320
$19.34 \pm 0.43$

$32.70 \pm 0.40$

$35.03 \pm 0.41$

$43.62 \pm 0.38$

$54.67 \pm 0.33$
$\mathrm{Cu}_{2} \mathrm{Zn}_{0.5} \mathrm{Sn}_{0.9} \mathrm{~S}_{0.3} \mathrm{Se}_{2.7}$

$\mathrm{Cu}_{2} \mathrm{Zn}_{0.6} \mathrm{Sn}_{0.9} \mathrm{~S}_{0.3} \mathrm{Se}_{2.8}$
Estimated band-gap (eV)

1.0
1.1
1.1
1.1
1.2


nanocrsytals present here provide a structure model for studying the possible carrier confinement and spatial charger separation in polytypic $\mathrm{I}_{2}-\mathrm{II}-\mathrm{IV}-\mathrm{VI}_{4}$ nanocrystals. Noting that transformation between different polymorphs could be triggered through proper thermal activation, the polytypic nanocrystals may also be a structure model to investigate the effect of the pre-existed uniform interface on transformation.

\section{Methods}

Synthesis of polytypic CZTSSe nanocrystals with different phase ratio. $0.28 \mathrm{mmol}$ CuI, $0.14 \mathrm{mmol} \mathrm{Zn}\left(\mathrm{CH}_{3} \mathrm{COO}\right)_{2} \cdot 2 \mathrm{H}_{2} \mathrm{O}$ and $0.14 \mathrm{mmol} \mathrm{SnCl}{ }_{2} \cdot 2 \mathrm{H}_{2} \mathrm{O}$ were dissolved in $10 \mathrm{~mL}$ oleylamine with the presence of $600 \mu \mathrm{L}$ 1-dodecanethiol in a three-neck flask in air and then heated up to $180^{\circ} \mathrm{C}$. At the same time, $1.12 \mathrm{mmol}$ diphenyl diselenide was dissolved in another three-neck flask containing $5 \mathrm{~mL}$ oleylamine in air at $70^{\circ} \mathrm{C}$. Reaction solutions were mixed by injecting diphenyl diselenide solution into the former salt solution. The temperature of the reaction solution was increased from $150^{\circ} \mathrm{C}$ to $280^{\circ} \mathrm{C}$ at a heating rate of $10^{\circ} \mathrm{C} / \mathrm{min}$ and kept at $280^{\circ} \mathrm{C}$ for $1 \mathrm{~h}$. To synthesize polytypic CZTSSe nanocrystals with different phase ratios, the reaction temperatures were kept at $240,260,300$ and $320^{\circ} \mathrm{C}$, respectively, while all other reaction parameters were kept the same.

Synthesis of polytypic CCTSSe nanocrystals. The synthesis procedure of polytypic CCTSSe nanocrystals is similar to that of CZTSSe polytypic nanocrystals except $0.14 \mathrm{mmol} \mathrm{Zn}\left(\mathrm{CH}_{3} \mathrm{COO}\right)_{2} \cdot 2 \mathrm{H}_{2} \mathrm{O}$ was then substituted by $0.14 \mathrm{mmol}$ $\mathrm{Cd}\left(\mathrm{CH}_{3} \mathrm{COO}\right)_{2} \cdot 2 \mathrm{H}_{2} \mathrm{O}$ while other reaction parameters were kept the same as with CZTSSe. The reaction was held at $280^{\circ} \mathrm{C}$ for $1 \mathrm{~h}$.

When the reaction completed, the flask was removed from the heating mantle and naturally cooled down. The black product was collected and centrifuged at $8000 \mathrm{rpm}$ for $5 \mathrm{~min}$ and the upper clear solution was discarded. Then hexane was added to disperse the nanocrystals. The dispersion was centrifuged at $5000 \mathrm{rpm}$ for $10 \mathrm{~s}$, larger nanocrystals and aggregates were discarded while the bright black colloidal solution was transferred into another centrifuge tube where slurries formed after the addition of ethanol. Those slurries were centrifuged again at $8000 \mathrm{rpm}$ for $5 \mathrm{~min}$ and the nanocrystals accumulated at the bottom of centrifuge tube once again, the washing process was repeated for two times. A typical reaction yields about $40 \mathrm{mg}$ dispersible nanocrystals

Measurement and characterization. The product was characterized by powder Xray diffraction, using a Philips X'Pert PRO SUPER X-ray diffractometer with graphite monochromated $\mathrm{Cu} \mathrm{K} \alpha$ radiation $(\lambda=1.54056 \AA)$. The operation voltage and current were kept at $40 \mathrm{kV}$ and $400 \mathrm{~mA}$, respectively. The phase purity and relationships were analyzed using the X'Pert High Score plus software in conjunction with the ICDD database. All structural refinements were carried out using the Rietveld refinement technique with the Fullprof software suite (version 2.05) ${ }^{41}$. For CZTSSe, the space groups were taken as $P 6_{3} m c$ and $F-43 m$. For CCTSSe, the space groups were taken as $P m n 2_{1}$ and $I-42 m$. Pseudo-Voigt profile functions were employed and 18 least-squares parameters (zero-point parameter, scale factor, lattice parameters, profile parameters $\mathrm{U}, \mathrm{V}, \mathrm{W}$, Shape, $\mathrm{X}$ and asymmetry) were varied during the refinement process.

Nanocrystals dispersed in hexane were dropped on carbon supported $\mathrm{Cu}$ or $\mathrm{Mo}$ grids for TEM and HRTEM observation, which were performed on JEOL-2010F with an acceleration voltage of $200 \mathrm{KV}$. We tilted the sample within angles of $\pm 15^{\circ}$ along the $\mathrm{x}$ and $\mathrm{y}$-axis during the TEM observation. EDS and STEM EDS element mapping were carried out on Inca Oxford equipped on JEOL-2010F, nylon grids were used to determine chemical compositions and Be grids were used for EDS mapping and line scan. To clarify whether 1-dodecanethiol makes the $\mathrm{S}$ quantification dubious, we deposited the nanocrystals on carbon supported $\mathrm{Cu}$ grids and heated the grids at $250^{\circ} \mathrm{C}$ (Naberther PHTO 80-450/15 tube furnace) to remove 1-dodecanethiol under vacuum $\left(-1\right.$ bar) for $0.5 \mathrm{~h}$, the heating and cooling rates were kept as $5{ }^{\circ} \mathrm{C} / \mathrm{min}$. Considering the boiling point of 1-dodecanethiol under ambient condition is $266 \sim 283^{\circ} \mathrm{C}$, it is reasonable to propose that the 1-dodecanethiol can be well removed after heating treatment at $250^{\circ} \mathrm{C}$ for $0.5 \mathrm{~h}$ under vacuum. We found that the $\mathrm{Zn}: \mathrm{Sn}: \mathrm{S}$ molar ratios of the same batch sample collected on nylon grids and $\mathrm{Cu}$ grids are almost unchanged (see Supplementary Table S2 and Fig. S3 online), indicating that sulfur signal originated from 1-dodecanethiol are not noticeable to affect the accuracy of sulfur quantification. Unexpectedly, we find Se/S molar ratios of all samples decrease after heating treatment, this can be understood by the fact that $\mathrm{Cu}_{2} \mathrm{ZnSnS}_{4}$ firstly losses $\mathrm{S}$ during heating ${ }^{42}$ and $\mathrm{Cu}_{2} \mathrm{ZnSnS}_{4}$ are more stable than $\mathrm{Cu}_{2} \mathrm{ZnSnSe}_{4}{ }^{43}$, as a result, CZTSSe alloy will firstly losses Se during heating treatment while the $\mathrm{Zn}: \mathrm{Sn}: \mathrm{S}$ molar ratio is unchanged. Optical absorption spectra of nanocrystals dispersed in hexane were measured at room temperature using a DUV-3700 ultraviolet-visible-near infrared (UV-vis-NIR) spectrometer (Shimadzu).

Crystal structure simulation. Theoretical calculations have shown that the tetragonal kesterite (space group I-4) and stannite (space group I-42m) structures are the low-energy ZB-derived structures, and the orthorhombic WZ-kesterite (space group $P c$ ) and WZ-stannite (space group $P m n 2_{1}$ ) are the low-energy WZ-derived structures ${ }^{39}$. When $\mathrm{Cu}, \mathrm{Zn}$ and $\mathrm{Sn}$ cations become totally disordered, the $\mathrm{ZB}$ - and WZ-derived quaternary structures are equivalent to the binary ZB and WZ structures, respectively. We simulated the PXRD patterns of (i) the ordered ZB-derived kesterite structure and WZ-derived WZ-kesterite structure of CZTSe, (ii) the disordered ZBand WZ-derived CZTSe structures. For the ordered structures, the atomic coordinates and the lattice parameters are determined from the first-principles structural relaxation ${ }^{39,44}$. For the disordered structures, the random distribution of $\mathrm{Cu}, \mathrm{Zn}$ and $\mathrm{Sn}$ makes them indistinguishable, and thus the structures possess the same space groups and atomic coordinates as the binary ZB $(F-43 m)$ and WZ $\left(\mathrm{Pb}_{3} \mathrm{mc}\right)$ structures. The lattice parameters of the disordered structures are derived assuming that the disordered structures have the same volume per cell as the ordered structures. For $\mathrm{Cu}_{2} \mathrm{CdSnSe}_{4}$ (CCTSe), as stannite and WZ-stannite CCTSe are the most stable ZB- and WZ-derived structures, respectively ${ }^{39}$, we simulated the PXRD patterns of (iii) ordered stannite and WZ-stannite, and (iv) disordered $\mathrm{ZB}$ and $\mathrm{WZ} \mathrm{Cu}_{2} \mathrm{CdSnSe}_{4}$ through a similar approach for comparison. The detailed parameters needed for the simulations of different polymorphs of CZTSe and CCTSe are given in Table S1 (see Supplementary).

1. Park, C. H., Cheong, B. H., Lee, K. H. \& Chang, K. J. Structural and electronicproperties of cubic 2H, 4H, and 6H SiC. Phys. Rev. B 49, 4485-4493 (1994).

2. Yeh, C. Y., Lu, Z. W., Froyen, S. \& Zunger, A. Zinc-blende-wurtzite polytypism in semiconductors. Phys. Rev. B 46, 10086-10097 (1992).

3. Murayama, M. \& Nakayama, T. Chemical trend of band offsets at wurtzite zinc blende heterocrystalline semiconductor interfaces. Phys. Rev. B 49, 4710-4724 (1994).

4. Ito, T. Simple criterion for wurtzite-zinc-blende polytypism in semiconductors. Jap. J. App. Phys. Part 2-Letters 37, L1217-L1220 (1998).

5. Glas, F., Harmand, J. C. \& Patriarche, G. Why does wurtzite form in nanowires of III-V zinc blende semiconductors? Phys. Rev. Lett. 99, 146101 (2007).

6. Jacobs, B. W. et al. Electronic and structural characteristics of zinc-blende wurtzite biphasic homostructure GaN nanowires. Nano Lett. 7, 1435-1438 (2007).

7. Zhou, F. et al. Thermal conductivity of indium arsenide nanowires with wurtzite and zinc blende phases. Phys. Rev. B 83, 205416 (2011).

8. Li, D., Wang, Z. \& Gao, F. First-principles study of the electronic properties of wurtzite, zinc-blende, and twinned InP nanowires. Nanotechnology 21, 505709 (2010).

9. Caroff, P. et al. Controlled polytypic and twin-plane superlattices in III-V nanowires. Nature Nanotech. 4, 50-55 (2009).

10. Caroff, P., Bolinsson, J. \& Johansson, J. Crystal phases in III-V nanowires: From random toward engineered polytypism. IEEE J Sel Top Quant 17, 829-846 (2011).

11. Algra, R. E. et al. Twinning superlattices in indium phosphide nanowires. Nature 456, 369-372 (2008)

12. Akiyama, T., Yamashita, T., Nakamura, K. \& Ito, T. Band alignment tuning in twin-plane superlattices of semiconductor nanowires. Nano Lett. 10, 4614-4618 (2010).

13. Wei, S. H., Zhang, S. B. \& Zunger, A. Band structure and stability of zinc-blendebased semiconductor polytypes. Phys. Rev. B 59, R2478-R2481 (1999).

14. Carbone, L. \& Cozzoli, P. D. Colloidal heterostructured nanocrystals: Synthesis and growth mechanisms. Nano Today 5, 449-493 (2010).

15. Donega, C. d. M. Synthesis and properties of colloidal heteronanocrystals. Chem. Soc. Rev. 40, 1512-1546 (2011).

16. Costi, R., Saunders, A. E. \& Banin, U. Colloidal hybrid nanostructures: A new type of functional materials. Angew. Chem.-Int. Edit. 49, 4878-4897 (2010).

17. Manna, L., Scher, E. C. \& Alivisatos, A. P. Synthesis of soluble and processable rod-, arrow-, teardrop-, and tetrapod-shaped CdSe nanocrystals. J. Am. Chem. Soc. 122, 12700-12706 (2000).

18. Peng, Z. A. \& Peng, X. G. Nearly monodisperse and shape-controlled CdSe nanocrystals via alternative routes: Nucleation and growth. J. Am. Chem. Soc. $\mathbf{1 2 4}$ 3343-3353 (2002).

19. Manna, L. et al. Controlled growth of tetrapod-branched inorganic nanocrystals. Nat. Mater. 2, 382-385 (2003).

20. Deka, S. et al. Octapod-shaped colloidal nanocrystals of cadmium chalcogenides via "One-Pot" cation exchange and seeded growth. Nano Lett. 10, 3770-3776 (2010).

21. Guo, Q. J., Hillhouse, H. W. \& Agrawal, R. Synthesis of $\mathrm{Cu}_{2} \mathrm{ZnSnS}_{4}$ nanocrystal ink and its use for solar cells. J. Am. Chem. Soc. 131, 11672-11673 (2009).

22. Riha, S. C., Parkinson, B. A. \& Prieto, A. L. Solution-based synthesis and characterization of $\mathrm{Cu}_{2} \mathrm{ZnSnS}_{4}$ nanocrystals. J. Am. Chem. Soc. 131, 12054-12055 (2009).

23. Steinhagen, C. et al. Synthesis of $\mathrm{Cu}_{2} \mathrm{ZnSnS}_{4}$ nanocrystals for use in low-cost photovoltaics. J. Am. Chem. Soc. 131, 12554-12555 (2009).

24. Shavel, A., Arbiol, J. \& Cabot, A. Synthesis of quaternary chalcogenide nanocrystals: stannite $\mathrm{Cu}_{2} \mathrm{Zn}_{\mathrm{x}} \mathrm{Sn}_{\mathrm{y}} \mathrm{Se}_{1+\mathrm{x}+2 \mathrm{y}}$. J. Am. Chem. Soc. 132, 4514-4515 (2010).

25. Riha, S. C., Parkinson, B. A. \& Prieto, A. L. Compositionally tunable $\mathrm{Cu}_{2} \mathrm{ZnSn}\left(\mathrm{S}_{1-\mathrm{x}} \mathrm{Se}_{\mathrm{x}}\right)_{4}$ nanocrystals: probing the effect of Se-inclusion in mixed chalcogenide thin films. J. Am. Chem. Soc. 133, 15272-15275 (2011).

26. Guo, Q. et al. Fabrication of 7.2\% efficient CZTSSe solar cells using CZTS Nanocrystals. J. Am. Chem. Soc. 132, 17384-17386 (2010).

27. Liu, M. L., Chen, I. W., Huang, F. Q. \& Chen, L. D. Improved thermoelectric properties of Cu-doped quaternary chalcogenides of $\mathrm{Cu}_{2} \mathrm{CdSnSe}_{4}$. Adv. Mater. 21, 3808-3812 (2009). 
28. Fan, F. J. et al. Colloidal synthesis of $\mathrm{Cu}_{2} \mathrm{CdSnSe}{ }_{4}$ nanocrytals and hot-pressing to enhance the thermoelectric figure-of-merit. J. Am. Chem. Soc. 133, 15910-15913 (2011).

29. Ibanez, M. et al. Composition control and thermoelectric properties of quaternary chalcogenide nanocrystals: The case of stannite $\mathrm{Cu}_{2} \mathrm{CdSnSe}_{4}$. Chem. Mater. 24, 562-570 (2012).

30. Ibáñez, M. et al. Extending the nanocrystal synthesis control to quaternary compositions. Cryst. Growth Des. 12, 1085-1090 (2012).

31. Pan, D. C. et al. Synthesis of Cu-In-S ternary nanocrystals with tunable structure and composition. J. Am. Chem. Soc. 130, 5620-5621 (2008).

32. Norako, M. E. \& Brutchey, R. L. Synthesis of metastable Wurtzite CuInSe ${ }_{2}$ nanocrystals. Chem. Mater. 22, 1613-1615 (2010).

33. Lu, X. T., Zhuang, Z. B., Peng, Q. \& Li, Y. D. Wurtzite $\mathrm{Cu}_{2} \mathrm{ZnSnS}_{4}$ nanocrystals: a novel quaternary semiconductor. Chem. Commun. 47, 3141-3143 (2011).

34. Wang, J. J., Hu, J. S., Guo, Y. G. \& Wan, L. J. Wurtzite $\mathrm{Cu}_{2} \mathrm{ZnSnSe}_{4}$ nanocrystals for high-performance organic-inorganic hybrid photodetectors. NPG Asia Materials 4, e2 (2012).

35. Koo, B., Patel, R. N. \& Korgel, B. A. Wurtzite-chalcopyrite polytypism in CuInS 2 nanodisks. Chem. Mater. 21, 1962-1966 (2009)

36. Xu, C. J., Wang, B. D. \& Sun, S. H. Dumbbell-like Au- $\mathrm{Fe}_{3} \mathrm{O}_{4}$ nanoparticles for target-specific platin delivery. J. Am. Chem. Soc. 131, 4216-4217 (2009).

37. Fan, F. J. et al. Large-scale colloidal synthesis of non-stoichiometric $\mathrm{Cu}_{2} \mathrm{ZnSnSe}_{4}$ nanocrystals for thermoelectric applications. Adv. Mater. 24, 6158-6163 (2012).

38. Spirkoska, D. et al. Structural and optical properties of high quality zinc-blende/ wurtzite GaAs nanowire heterostructures. Phys. Rev. B 80, 245325 (2009).

39. Chen, S. Y. et al. Wurtzite-derived polytypes of kesterite and stannite quaternary chalcogenide semiconductors. Phys. Rev. B 82, 195203 (2010).

40. Wei, S. H. \& Zhang, S. B. Structure stability and carrier localization in CdX (X $=$ S Se, Te) semiconductors. Phys. Rev. B 62, 6944-6947 (2000).

41. Rodríguez-Carvajal, J. Recent advances in magnetic structure determination by neutron powder diffraction. Physica B: Condensed Matter 192, 55-69 (1993).

42. Scragg, J. J. et al. Chemical isights into the instability of $\mathrm{Cu}_{2} \mathrm{ZnSnS}_{4}$ films during annealing. Chem. Mater. 23, 4625-4633 (2011).

43. Maeda, T., Nakamura, S. \& Wada, T. Phase stability and electronic structure of InFree photovoltaic semiconductors, $\mathrm{Cu}_{2} \mathrm{ZnSnSe}_{4}$ and $\mathrm{Cu}_{2} \mathrm{ZnSnS}_{4}$ by firstprinciples calculation. Thin-Film Compound Semiconductor Voltaics-2009 1165, 137-143 (2010).
44. Chen, S. Y., Gong, X. G., Walsh, A. \& Wei, S. H. Electronic structure and stability of quaternary chalcogenide semiconductors derived from cation crosssubstitution of II-VI and I-III-VI 2 compounds. Phys. Rev. B 79, 165211 (2009).

\section{Acknowledgments}

S.-H.Y. acknowledges the funding support from the National Basic Research Program of China (Grant 2010CB934700), the National Natural Science Foundation of China (Grants 91022032, 912271032, 21061160492, J1030412), the Chinese Academy of Sciences (Grant KJZD-EW-M01-1), the Ministry of Science and Technology of China (2012BAD32B05-4), the International Science \& Technology Cooperation Program of China (Grant 2010DFA41170), the Principal Investigator Award by the National Synchrotron Radiation Laboratory at the University of Science and Technology of China. S. Chen is supported by NNSEC (Grant 61106087). This work is also supported by CAS and USTC Special Grants for Postgraduate Research, Innovation and Practice. We thank Prof. Kai-Bing Tang at the University of Science and Technology of China for helpful discussions; we also thank Dr. Bo $\mathrm{Yu}$ at the Boston College for help during the manuscript preparation.

\section{Author contributions}

F.J.F. planned and performed the experiments, collected and analyzed the data, and wrote the paper. S.H.Y. supervised the project, and conceived the experiments, analyzed the results and wrote the paper. L.W., H.B.Y., H.W.L., Y.X.W. helped with synthesis of the materials and collected the data. M.G. did the elemental mapping. S.Y.C. did the modeling work. G.Y.L. did the Rietveld fit of the obtained polytypic nanocrystals. All authors discussed the results and commented on the manuscript.

\section{Additional information}

Supplementary information accompanies this paper at http://www.nature.com/ scientificreports

Competing financial interests: The authors declare no competing financial interests. License: This work is licensed under a Creative Commons Attribution-NonCommercial-NoDerivs 3.0 Unported License. To view a copy of this license, visit http://creativecommons.org/licenses/by-nc-nd/3.0/

How to cite this article: Fan, F. et al. Linearly arranged polytypic CZTSSe nanocrystals. Sci. Rep. 2, 952; DOI:10.1038/srep00952 (2012). 